Article

\title{
Neutrosophic Components Semigroups and Multiset Neutrosophic Components Semigroups
}

\author{
Vasantha W. B. ${ }^{1}\left(\mathbb{D}\right.$, Ilanthenral Kandasamy ${ }^{1, *(1)}$ and Florentin Smarandache ${ }^{2}(\mathbb{D})$ \\ 1 School of Computer Science and Engineering, VIT, Vellore, Tamilnadu 632014, India; vasantha.wb@vit.ac.in \\ 2 Department of Mathematics, University of New Mexico, Gallup Campus, NM 87131, USA; \\ smarand@unm.edu \\ * Correspondence: ilanthenral.k@vit.ac.in
}

Received: 15 April 2020; Accepted: 11 May 2020; Published: 16 May 2020

\begin{abstract}
Neutrosophic components (NC) under addition and product form different algebraic structures over different intervals. In this paper authors for the first time define the usual product and sum operations on NC. Here four different NC are defined using the four different intervals: $(0,1),[0,1),(0,1]$ and $[0,1]$. In the neutrosophic components we assume the truth value or the false value or the indeterminate value to be from the intervals $(0,1)$ or $[0,1)$ or $(0,1]$ or $[0,1]$. All the operations defined on these neutrosophic components on the four intervals are symmetric. In all the four cases the $\mathrm{NC}$ collection happens to be a semigroup under product. All of them are torsion free semigroups or weakly torsion free semigroups. The NC defined on the interval $[0,1)$ happens to be a group under addition modulo 1 . Further it is proved the NC defined on the interval $[0,1)$ is an infinite commutative ring under addition modulo 1 and usual product with infinite number of zero divisors and the ring has no unit element. We define multiset NC semigroup using the four intervals. Finally, we define n-multiplicity multiset NC semigroup for finite $n$ and these two structures are semigroups under + modulo 1 and $\{M(S),+, \times\}$ and $\{n-M(S),+, \times\}$ are NC multiset semirings. Several interesting properties are discussed about these structures.
\end{abstract}

Keywords: neutrosophic components (NC); NC semigroup; multiset NC; n-multiplicity; multiset NC semigroup; special zero divisors; torsion free semigroup; weakly torsion free semigroup; infinite commutative ring; group under addition modulo 1; infinite neutrosophic communicative ring; multiset NC semirings

\section{Introduction}

Semigroups play a vital role in algebraic structures [1-5] and they are applied in several fields and it is a generalization of groups, as all groups are semigroups and not vice versa. Neutrosophic sets proposed by Smarandache in [6] has become an interesting area of major research in recent days both in the area of algebraic structures [7-11] as well as in applications ranging from medical diagnosis to sentiment analysis [12,13]. The study of neutrosophic triplets happens to be a special form of neutrosophic sets. Extensive study in this direction have been carried out by several researchers in $[8,14-17]$. Here we are interested in the study of neutrosophic components (NC) over the intervals $(0,1),(0,1],[0,1)$ and $[0,1]$. So far researchers have studied and applied NC only on the interval $[0,1]$ though they were basically defined by Smarandache [18] on all intervals. Further they have not studied them under the usual operation + and $\times$. Here we venture to study NC on all the four intervals and obtain several interesting algebraic properties about them.

Smarandache multiset semigroup studied in [19] is different from these semigroups. Further these multiset NC semigroups are also different from multi semigroups in [20] which deals with multi structures on semigroups. 
Any algebraic structure becomes more efficient for application only when it enjoys some strong properties. In fact a set endowed with closed associative binary operation happens to be a semigroup. This semigroup structure does not yield many applications like algebraic codes or commutative rings or commutative semirings. Basically to have a vector space one needs at least the basic algebraic structure to be a group under addition. The same is true in case of algebraic codes. However none of the intervals $[0,1]$ or $(0,1)$ or $(0,1]$ can afford to have a group structure under + . One can not imagine of a group structure under product for no inverse element can be got for any element in these intervals. But when we consider the interval $[0,1)$ we see it is a group under addition modulo 1 .

In fact for any collection of NC which are triplets to have a stronger structure than a semigroup we need to have a strong structure on the interval over which it is built. That is why this paper studies the NC on the interval $[0,1)$. These commutative rings in $[0,1)$ can be used to built both algebraic codes on the NC for which we basically need these NC to be at least a commutative ring. With this motivation, we have developed this paper.

This paper further proves that multiset NC built on the interval $[0,1)$ happens to be a commutative semiring paving way to build multiset NC algebraic codes and multiset neutrosophic algebraic codes which can be applied to cryptography with indeterminacy.

The paper is organized as follows. Section one is introductory in nature. Section 2 recalls the basic concepts of partial order, torsion free semigroup and neutrosophic set. Section 3 introduces NC on the four intervals $[0,1],(0,1),[0,1)$ and $(0,1]$ and mainly prove they are infinite NC semigroups which are torsion free. The new notion of weakly torsion free elements in a semigroup is introduced in this paper and it is proved that NC semigroups built on intervals $[0,1]$ and $[0,1)$ are weakly torsion free under usual product $\times$. We further prove the NC built using the interval $[0,1)$ happens to be an infinite order commutative ring with infinite number of zero divisors and it has no unit. In Section 4 we prove multiset NC built using these four intervals are multiset neutrosophic semigroups under usual product $\times$. We prove only in case of $[0,1)$ the multiset NC is a ring with infinite number of zero divisors and in all the other interval, $M(S)$ is a torsion free or weakly torsion free semigroup under $\times$. Only in case of the interval $[0,1), M(S)$ is semigroup under modulo addition 1 . In Section 5 we define $\mathrm{n}$-multiplicity multiset NC on all the intervals and obtain several interesting properties. Discussions about this study are given in Section 6 and the final section gives conclusions and future research based on their structures.

\section{Basic Concepts}

In this section we introduce the basic concepts needed to make this paper a self contained one. We first recall the definition of partially ordered set.

Definition 1. There exist some distinct elements $a, b \in S$ such that $a<b$ or $a>b$, and other distinct elements $b, c \in S$ such that neither $b<c$ nor $b>c$, then we say $(S,<)$ is a partially ordered set. We say $(S, \leq)$ is a totally ordered set if for every pair $a, b, \in S$ we have $a \leq b$ or $b \geq a$.

The set of integers is a totally ordered set and the power set of a set $X ; P(X)$ is only a partially ordered set.

Next we proceed on to define torsion free semigroup.

Definition 2. A semigroup $\{S, \times\}$ is said to be a torsion free semigroup if for $a, b \in S, a \neq b, a^{n} \neq b^{n}$ for any $1 \leq n<\infty$.

We recall the definition of semiring in the following from [21].

Definition 3. For a non empty set $S,\{S,+, \times\}$ is defined as a semiring if the following conditions are true

1. $\{S,+\}$ is a commutative semigroup with 0 as its additive identity.

2. $\{S, \times\}$ is a semigroup. 
3. $a \times(b+c)=a \times b+a \times c$ for all $a, b, c, \in S$ follows distribution law.

If $\{S, \times\}$ is a commutative semigroup we call $\{S,+, \times\}$ as a commutative semiring.

For more, see [21].

For example, set of integers under product is a torsion free semigroup. Finally we give the basic definition of neutrosophic set.

Definition 4. The Neutrosophic components (NC) is a triplet $(a, b, c)$ where $a$ is the truth membership function from the unit interval $[0,1], b$ is the indeterminacy membership function and $c$ is the falsity membership function all of them are from the unit interval $[0,1]$.

For more about Neutrosophic components (NC), sets and their properties please refer [6].

Next we proceed onto define the notion of multiset.

Definition 5. A neutrosophic multiset is a neutrosophic set where one or more elements are repeated with same neutrosophic components or with different neutrosophic components.

Example 1. $M=\{a(0.3,0.4,0.5), a(0.3,0.4,0.5), b(1,0,0.2), b(1,0,0.2), c(0.7,1,0)\}$ is a neutrosophic multiset. For more refer [18]. However we in this paper use the term multiset NC to denote elements of the form $\{5(0.3,0.4,1), 3(0.6,0,1),(0,0.7,0.5)\}$ so 5 is the multiplicity of the NC $(0.3,0.4,1)$ and 3 is the multiplicity of the NC $(0.6,0,1)$ and 1 is the multiplicity of the NC $(0,0.7,0.5)$.

For more about multisets and multiset graphs [18,22].

\section{Neutrosophic Components (NC) Semigroups under Usual Product and Sum}

Throughout this section $\{x, y, z\}$ will denote the truth value, indeterminate value, false value where $x, y, z$ belongs to $[0,1]$, the neutrosophic set. However we define special NC on the intervals $(0,1),(0,1]$ and $[0,1)$. We first prove $S_{1}=\{(x, y, z) / x, y, z \in(0,1)\}$ is a semigroup under product and obtain several interesting properties about NC semigroups using the four intervals $(0,1),(0,1],[0,1)$ and $[0,1]$.

Example 2. Let $a=(0.3,0.8,0.5)$ and $b=(0.9,0.2,0.7)$ be any two NC in $S_{1}$. We define product $a \times b=$ $(0.3,0.8,0.5) \times(0.9,0.2,0.7)=(0.3 \times 0.9,0.8 \times 0.2,0.5 \times 0.7)=(0.27,0.16,0.35)$. It is again a neutrosophic set in $S_{1}$.

Definition 6. The four NC $S_{1}=\{(x, y, z) / x, y, z \in(0,1)\}, S_{2}=\{(x, y, z) / x, y, z \in[0,1)\}, S_{3}=$ $\{(x, y, z) / x, y, z \in(0,1]\}$ and $S_{4}=\{(x, y, z) / x, y, z \in[0,1]\}$ are all only partially ordered sets for if $a=(x$, $y, z)$ and $b=(s, r, t)$ are in $S_{i}$ then $a<b$ if and only if $x<s, y<r, z<t$; but not all elements are ordered in $S_{i}$, that is why we say $S_{i}$ are only partially ordered sets, and denote it by $\left(S_{i}, \leq\right)$; where $\leq$ denotes the classical order relation over reals; $1 \leq i \leq 4$.

For instance if $a=(0.3,0.7,0.5)$ and $b=(0.5,0.2,0.3)$ are in $S_{i}$ then $a$ and $b$ cannot be compared. If $d=(0.8,0.5,0.7)$ and $c=(0.6,0.2,0.5)$, then $d>c$ or $c<d$.

In view of this we have the following theorem.

Theorem 1. Let $S_{1}=\{(x, y, z) / x, y, z \in(0,1)\}$ be the collection of all NC which are such that the elements $x, y$ and $z$ do not take any extreme values.

1. $\left\{S_{1}, \times\right\}$ is an infinite order commutative semigroup which is not a monoid and has no zero divisors.

2. Every $a=(x, y, z)$ in $S_{1}$ will generate an infinite cyclic subsemigroup under product of $S_{1}$ denoted by $(P, \times)$. 
3. The elements of $P$ forms a totally ordered set, (for if $a=(x, y, z) \in P$ we see $a^{2}=a \times a<a$ ).

4. $\left\{S_{1}, \times\right\}$ has no idempotents and $\left\{S_{1}, \times\right\}$ is a torsion free semigroup.

Proof. Proof of 1: Clearly if $a=(x, y, z)$ and $b=(r, s, t)$ are in $S_{1}$, then $a \times b=(x \times r, y \times s, z \times t)$ is in $S_{1}$; as $x \times r, y \times s$ and $z \times t \in(0,1)$. Hence, $\left\{S_{1}, \times\right\}$ is a semigroup under product. Further as number of elements in $(0,1)$ is infinite so is $S_{1}$. Finally as the product in $(0,1)$ is commutative so is the product in $S_{1}$. Hence the claim. $(1,1,1)$ is not in $S_{1}$ as we have used only the open interval $(0,1)$, we see $\left\{S_{1}, \times\right\}$ is not a monoid. $S_{1}$ has no zero divisors as the elements are from the open interval which does not include 0 , hence the claim.

Proof of 2: Let $a=(x, y, z)$ be in $S$, we see $a \times a=(x \times x, y \times y, z \times z)=a^{2}$, and so on $a \times a \times \ldots \times a=a^{n}=\left(x^{n}, y^{n}, z^{n}\right)$ and $n$ can take values from $(0, \infty)$. Thus $a$ in $S$ generates a cyclic subsemigroup of infinite order, hence the claim.

Proof of 3: Let $P=\langle a\rangle, a$ generates the semigroup under product, it is of infinite order and from the property of elements in $(0,1) ; a>a^{2}>a^{3}>$ and so on $>a^{n}$. Hence the claim.

Proof of 4: If any $a=(x, y, z) \in S_{1}$ as $x, y, z \in(0,1)$, and $\mathrm{x}, \mathrm{y}$ and $\mathrm{z}$ are torsion free so is $a$. We see $a^{2} \neq a$ for any $a \in S_{1}$. Further if $a \neq b$ for no $n \in(0, \infty) ; a^{n}=b^{n}$. Hence the claim.

Definition 7. The four NC $S_{1}, S_{2}, S_{3}$ and $S_{4}$ mentioned in definition 6 under the usual product $\times$ forms a commutative semigroup of infinite order defined as the NC semigroups.

Theorem 2. Let $S_{2}=\{(x, y, z) / x, y, z \in[0,1)\}$ be the collection of NC. $\left\{S_{2}, \times\right\}$ is only a semigroup and not a monoid and has infinite number of zero divisors. Further all other results mentioned in Theorem 1 are true with an additional property if $a \neq b ;\left(a, b \in S_{2}\right)$ we have

$$
\lim _{n \rightarrow \infty} a^{n}=\lim _{n \rightarrow \infty} b^{n}=(0,0,0)
$$

as $(0,0,0) \in S_{2}$.

Proof as in case of Theorem 1.

In view of this we define an infinite torsion free semigroup to be weakly torsion free if $a \neq b$; but

$$
\lim _{n \rightarrow \infty} a^{n}=\lim _{n \rightarrow \infty} b^{n}
$$

Thus $S_{2}$ is only a weakly torsion free semigroup.

It is interesting to note $S_{1}$ is contained in $S_{2}$ and in fact $S_{1}$ is a subsemigroup of $S_{2}$.The differences between $S_{1}$ and $S_{2}$ is that $S_{2}$ has infinite number of zero divisors and the $\lim _{n \rightarrow \infty} a^{n}=(0,0,0)$ exists in $S_{2}$ and $S_{1}$ is torsion free but $S_{2}$ is weakly torsion free.

Theorem 3. Let $S_{3}=\{(x, y, z) / x, y, z \in(0,1]\}$ be the collection of NC. $\left\{S_{3}, \times\right\}$ is a monoid and has no zero divisors.

Results 2 to 4 of Theorem 1 are true. Finally $S_{1}$ is a subset of $S_{3}$, in fact $S_{1}$ is a subsemigroup of $S_{3}$. The main difference between $S_{1}$ and $S_{3}$ is that $S_{3}$ is a monoid and $S_{1}$ is not a monoid. The difference between $S_{2}$ and $S_{3}$ is that $S_{3}$ has no zero divisors but $S_{2}$ has zero divisors and $S_{3}$ is a monoid.

Next we prove a theorem for $S_{4}$.

Theorem 4. Let $S_{4}=\{(x, y, z) / x, y, z \in[0,1]\} .\left\{S_{4}, \times\right\}$ is a semigroup and is a monoid and has zero divisors. Other three conditions of Theorem 1 is true, but $S_{4}$ like $S_{2}$ is only a weakly torsion free semigroup.

Proof as in case of Theorem 1. We have $S_{1}$ contained in $S_{2}$ and $S_{2}$ is contained in $S_{4}$ and $S_{1}$ contained in $S_{3}$ and $S_{3}$ is contained in $S_{4}$. 
However, it is interesting to note $S_{2}$ and $S_{3}$ are not related in spite of the above relations.

Now we analyse all these four neutrosophic semigroups to find out, on which of them we can define addition modulo $1 . S_{1}$ does not include the element $(0,0,0)$ as 0 is not in $(0,1)$, so $S_{1}$ is not even closed under addition modulo 1 . So $S_{1}$ in not a semigroup or a group under plus modulo 1 . Since $S_{3}$ and $S_{4}$ contains $(1,1,1)$ we cannot define addition modulo 1 ; hence, they can not have any algebraic structure under addition modulo 1 . Now consider $\left\{S_{2},+\right\}$, clearly $\left\{S_{2},+\right\}$ is a group under addition modulo 1.

In view of all these we have the following theorem.

Definition 8. The NC $\left\{S_{2},+\right\}$ under usual addition modulo 1 is a group defined as the NC group denoted by $\left\{S_{2},+\right\}$.

Theorem 5. $\left\{S_{2},+\right\}$ is a group under addition modulo 1.

Proof. For any $y, x \in S_{2}, \mathrm{x}+\mathrm{y}(\bmod 1) \in S_{2} \cdot(0,0,0) \in S_{2}$ acts as additive identity. Further for every $x$ there is a unique $y \in S_{2}$ with $x+y=(0,0,0)$. Hence the theorem.

Definition 9. The NC $S_{2}$ under the operations of the usual addition + modulo 1 and usual product $\times$ forms a commutative ring of infinite order defined as the NC commutative ring denoted by $\left\{S_{2},+, \times\right\}$.

Theorem 6. $\left\{S_{2},+, \times\right\}$ is a commutative ring with infinite number of zero divisors and has no multiplicative identity $(1,1,1)$.

Proof. Follows from the Theorem 1 and the fact $S_{2}$ is closed under + modulo 1 by Theorem 5 . The distributive property is inherited from the number theoretic properties of modulo integers. As 1 is not in $[0,1) ;(1,1,1)$ is not in $S_{2}$, hence the result.

Next we proceed on to define multiset NC semigroups in the following section.

\section{Multiset NC Semigroups}

In this section we proceed on to define multiset NC semigroups using $S_{1}, S_{2}, S_{3}$ and $S_{4}$. We see $M\left(S_{1}\right)=\left\{\right.$ Collection of all multiset NC using elements of $\left.S_{1}\right\}$. On similar lines we define $M\left(S_{2}\right), M\left(S_{3}\right)$ and $M\left(S_{4}\right)$ using $S_{2}, S_{3}$ and $S_{4}$ respectively. We prove $\left\{M\left(S_{2}\right),+, \times\right\}$ is a multiset neutrosophic semiring of infinite order.

Recall [18], $A$ is a multi neutrosophic set, then $A=\{5(0.3,0.7,0.9), 12(0.6 .0 .2,0.7), 8(0.1,0.5,0.1)$, $(0.6,0.7,0.5)\}$; that is in the multiset neutrosophic set $A ;(0.3,0.7,0.9)$ has occurred 5 times; $(0.6,0.2$, $0.7)$ has occurred 12 times or its multiplicity is 12 in $A$ and so on.

Let $M\left(S_{1}\right)=\left\{\right.$ Collection of all multisets using the elements from $\left.S_{1}\right\}, M\left(S_{1}\right)$ is an infinite collection. We just show how the classical product is defined on $M\left(S_{1}\right)$.

Let $A=\{9(0.3,0.2,0.4), 2(0.6,0.7,0.1),(0.1,0.3,0.2)\}$ and $B=\{5(0.1,0.2,0.5), 10(0.8,0.4,0.5)\}$ in $M\left(S_{1}\right)$ be any two multisets. We define the classical product $\times$ of $A$ and $B$ as follows;

$$
\begin{array}{r}
A \times B=\{9(0.3,0.2,0.4) \times 5(0.1,0.2,0.5), 9(0.3,0.2,0.4) \times 10(0.8,0.4,0.5), \\
2(0.6,0.7,0.1) \times 5(0.1,0.2,0.5), 2(0.6,0.7,0.1) \times 10(0.8,0.4,0.5), \\
(0.1,0.3,0.2) \times 5(0.1,0.2,0.5),(0.1,0.2,0.5) \times 10(0.8,0.4,0.5)\} \\
=\{45(0.03,0.04,0.2), 90(0.24,0.08,0.2), 10(0.06,0.14,0.05), \\
20(0.48,0.28,0.05), 5(0.01,0.06,0.1), 10(0.08,0.08,0.25)\}
\end{array}
$$

$A \times B$ is in $M\left(S_{1}\right)$, thus $\left\{M\left(S_{1}\right), \times\right\}$ is a commutative semigroup of infinite order defined as the multiset NC semigroup. 
Definition 10. Let $M\left(S_{i}\right)$ be the multi NC using elements of $S_{i}(i=1,2,3,4),\left\{M\left(S_{i}\right), \times\right\}$ on the usual product $\times$ is defined as the multiset neutrosophic semigroup for $i=1,2,3$ and 4 .

Definition 11. Let $\left\{S_{2}, \times\right\}$ be the multiset NC semigroup under $\times$, elements of the form $(a, 0,0),(0, b, c)$ and so on which are infinite in number with $a, b, c \in S_{2}$ contribute to zero divisors. Hence multisets using these types of elements contribute to zeros of the form $n(0,0,0) ; 1<n<\infty$. As the zeros are of varying multiplicity we call these zero divisors as special type of zero divisors.

We will provide examples of them.

Example 3. Let $R=\left\{\left(S_{2}\right), \times\right\}$ be the multiset $N C$ semigroup under product. Let $A=(0.6,0,0)$ and $B=$ $(0,0.4,0.5)$ be in $R, A \times B=(0,0,0)$. Take $D=\{9(0.6,0.9,0)\}$ and $E=9(0,0,0.4)$ in $R$; we get $D \times E=$ $\{81(0,0,0)\}$. Take $W=\{7(0,0.5,0), 4(0,0.6,0)\}$ and $V=\{(0.7,0,0.4), 20(0.8,0,0)\}$ be two multisets in $R$; $W \times V=\{7 \times 44(0,0,0)+7 \times 20(0,0,0)+4 \times 44(0,0,0)+4 \times 20(0,0,0)\}=\{704(0,0,0)\}$ is a special type of zero divisor of $R$.

Thus $M\left(S_{2}\right)$ is closed under the binary operation $\times$.

Theorem 7. The neutrosophic multiset semigroups $\left\{M\left(S_{i}\right), \times\right\}$ for $i=1,2,3,4$ are commutative and of infinite order satisfying, the following properties for each $M\left(S_{i}\right) ; i=1,2,3,4$.

1. $\left\{M\left(S_{1}\right), \times\right\}$ has no trivial or non-trivial special type of zero divisors and no trivial or non-trivial idempotents.

2. $\left\{M\left(S_{2}\right), \times\right\}$ has infinite number of special type of zero divisors and no non-trivial idempotents.

3. $\left\{M\left(S_{3}\right), \times\right\}$ has no trivial or non-trivial special zero divisors but has $(1,1,1)$ as identity and has no non trivial idempotents.

4. $\left\{M\left(S_{4}\right), \times\right\}$ has non-trivial special type of zero divisors and has $(1,1,1)$ as its identity and has idempotents of the form $\{(0,1,0),(1,1,0),(0,0,1),(1,0,1)$ and so on $\}$.

Proof. 1. Follows from the fact that $S_{1}$ has no zero divisors and idempotents as it is built on the interval $(0,1)$.

2. Evident from the fact $S_{2}$ is built on $[0,1)$ so has special type of zero divisors by definition but no idempotent.

3. True from the fact $S_{3}$ is built on $(0,1]$, so $(1,1,1) \in M\left(S_{3}\right)$.

4. $S_{4}$ which is built on $[0,1]$ has infinite special type of zero divisors as $(0,0,0) \in S_{4}$ by Definition 11 and $(1,1,1) \in M\left(S_{4}\right)$ and has idempotents of the form $\{(0,1,0),(1,1,0),(0,0,1),(1,0,1)$ and so on $\}$.

Hence the claims of the theorem.

Now we proceed onto define usual addition on $M\left(S_{1}\right)$

$S_{1}=\{(x, y, z) / x, y, z \in(0,1)\}$ in not even closed under addition. For there are $x, y \in(0,1)$ such that $x+y$ is 1 or greater than 1 , so these elements are not in $(0,1)$, hence our claim.

Recall $S_{2}=\{(x, y, z) / x, y, z \in[0,1)\}$. We can define addition modulo 1 and product under that addition both $S_{2}$ and $[0,1)$ are closed.

Let $a=(0.7,0.6,0.9)$ and $b=(0.5,0.9,0.4)$ be in $S_{2}$, we find $a+b \bmod 1$.

$a+b=(0.7,0.6,0.9)+(0.5,0.9,0.4)=(0.7+0.5(\bmod 1), 0.6+0.9(\bmod 1), 0.9+0.4(\bmod 1))=$ $(0.2,0.5,0.3)$ is in $S_{2} .(0,0,0)$ in $S_{2}$ acts as the additive identity.

For every $a \in S_{2}$ there is a unique $b \in S_{2}$ such that $a+b=(0,0,0) \bmod 1$. Thus $\left(S_{2},+\right)$ is a NC group of infinite under addition modulo 1 . Further $\left(S_{2}, \times\right)$ is a semigroup under product of infinite order which is commutative and not a monoid as $(1,1,1)$ is not in $S_{2}$.

Now we illustrate how addition is performed on any two neutrosophic multisets in $M\left(S_{2}\right)$.

Let $A=\{7(0.3,0.8,0.45), 9(0.02,0.41,0.9),(0.6,0.3,0.2)\}$ and $B=\{5(0.1,0,0.9), 2(0.6,0.5,0)\}$ be any two multisets of $M\left(S_{2}\right)$. To find the sum of $A$ with $B$ under addition modulo 1 . 
$A+B=\{35[(0.3,0.8,0.45)+(0.1,0,0.9)] \bmod 1,45[(0.02,0.41,0.9)+(0.1,0,0.9)] \bmod 1,5[(0.6,0.3$, $0.2)+(0.1,0,0.9)] \bmod 1,14[(0.3,0.8,0.45)+(0.6,0.5,0)] \bmod 1,18[(0.02,0.41,0.9)+(0.6,0.5,0)] \bmod 1$, $2[(0.6,0.3,0.2)+(0.6,0.5,0)] \bmod 1\}=\{35(0.4,0.8,0.35), 45(0.12,0.41,0.8), 5(0.7,0.3,0.1), 14(0.9,0.3$, $0.45), 18(0.62,0.91,0.9), 2(0.2,0.8,0.2)\}$

is in $M\left(S_{2}\right)$. This is the way addition modulo 1 operation is performed. For $M\left(S_{3}\right)$ and $M\left(S_{4}\right)$ we can not define usual addition modulo 1 as $(1,1,1) \in M\left(S_{3}\right)$ and $M\left(S_{4}\right)$.

Next we proceed on to describe the product of any two elements in $M\left(S_{2}\right)$. We take the above $A$ and $B$ and find $A \times B . A \times B=\{35[(0.3,0.8,0.45) \times(0.1,0,0.9)], 45[(0.02,0.41,0.9) \times(0.1,0,0.9)], 5[(0.6$, $0.3,02) \times(0.1,0,0.9)], 14[(0.3,0.8,0.45) \times(0.6,0.50)], 18[(0.02,0.41 .0 .9) \times(0.0 .6,0.5,0)], 2[(0.6,0.3,0.2)$ $\times(0.6,0.5,0)]\}=\{35(0.03,0,0.405), 45(0.002,0,0.81), 5(0.06,0,0.18), 14(0.18,0.4,0), 18(0.012,0.205,0)$, $2(0.36,0.15,0)\}$, is in $M\left(S_{2}\right)$.

Theorem 8. $\left\{M\left(S_{2}\right),+\right\}$ is a multiset NC semigroup under addition modulo 1.

Proof. $M\left(S_{2}\right)$ is closed under the binary operation addition modulo 1 . Thus $M\left(S_{2}\right)$ is the neutrosophic multiset semigroup under + modulo 1 .

Now we proceed on to define a special type of zero divisors. In view of this we have the following theorem.

Theorem 9. $R=\left\{M\left(S_{2}\right), \times\right\}$ is an infinite commutative multiset NC semigroup, which is not a monoid and has special type of zero divisors.

Proof. We see $M\left(S_{2}\right)$ under the binary operation product is closed and is associative as the base set $S_{2}$ is associative and commutative and is closed under the binary operation product. Thus $\left\{\left(S_{2}\right), \times\right\}$ is commutative semigroup of infinite order. Further $M\left(S_{2}\right)$ does not contain $(1,1,1)$ so $\left\{M\left(S_{2}\right), \times\right\}$ is not a monoid.

From the above definition and description of special zero divisors $R$ has infinite number of them.

We have the following theorem.

Theorem 10. $\left\{M\left(S_{2}\right),+, \times\right\}$ is a NC multiset commutative semiring of infinite order which has infinite numbers of special type of zero divisors.

Proof. Follows from Theorem 8 and Theorem 9.

Next we proceed on to define n- multiplicity neutrosophic multisets and derive some properties related with them. $M\left(S_{3}\right)$ and $M\left(S_{4}\right)$ are just multiset NC semigroups under product and in fact they are monoids. Further $M\left(S_{4}\right)$ has infinite number of special zero divisors.

\section{5. n-Multiplicity Neutrosophic Set Semigroups Using $S_{1}, S_{2}, S_{3}$ and $S_{4}$}

In this section we define the new notion of n-multiplicity NC using $S_{1}, S_{2}, S_{3}$ and $S_{4}$. We prove these n-multiplicity NC are of infinite order but what is restricted is the multiplicity n, that is any element cannot exceed multiplicity $n$; it can maximum be $n$, where $n$ is a positive finite integer. Finally we prove $\left\{M\left(S_{2}\right),+, \times\right\}$ where $S_{2}=[0,1)$ is a NC n-multiset commutative semiring of infinite order.

We will first illustrate this situation by some examples before we make an abstract definition of them.

Example 4. Let $4-M\left(S_{1}\right)=\left\{\right.$ collection all multisets with entries from $S_{1}=\{(x, y, z) / x, y, z \in$ $(0,1)\}$, such that any element in $S_{1}$ can maximum repeat itself only four times $\}$. Here $n=$ $4, A=\{4(0.5,0.7,0.4), 3(0.1,0.9,0.7), 4(0.1,0.2,0.3), 4(0.7,0.8,0.4), 4(0.8,0.8,0.8), 2(0.9,0.9,0.9)$, 
$3(0.7,0.9,0.6),(0.6,0.1,0.1)\}$ be a 4-multiplicity multiset from $4-M\left(S_{1}\right)$. We see the $N C$ $(0.5,0.7,0.4),(0.1,0.2,0.3),(0.7,0.8,0.4)$ and $(0.8,0.8,0.8)$ have multiplicity four which is the highest multiplicity an element of $4-M\left(S_{1}\right)$ can have. The NC $(0.1,0.9,0.7)$ and $(0.7,0.9,0.6)$ have multiplicity 3. The multiplicity of $(0.9,0.9,0.9)$ is two and that of $(0.6,0.1,0.1)$ is one. Clearly $S_{1}$ does not contain the extreme values 0 and 1 as $S_{1}$ is built using the open interval $(0,1)$. However on $M\left(S_{1}\right)$ we can not define addition.

Thus 4-M(S $\left.S_{1}\right)$ can not have the operation of addition defined on it. Now we show how the operation $\times$ is defined on $4-M\left(S_{1}\right)$ for the some $A, B \in 4-M\left(S_{1}\right)$. Now

$$
\begin{array}{r}
A \times B=\{3(0.3,0.7,0.8), 2(0.5,0.9,0.6), 4(0.2,0.3,0.4)\} \times\{(0.1,0.3,0.7), 2(0.5,0.7,0.1)\} \\
=\{3(0.03,0.21,0.56), 2(0.05,0.27,0.42), 4(0.02,0.09,0.28), \\
6(0.15,0.49,0.08), 4(0.25,0.63,0.06), 8(0.1,0.21,0.04)\}
\end{array}
$$

we now use the fact we can have maximum only 4 multiplicity of an element so we replace $6(0.15,0.49,0.08)$ by $4(0.15,0.49,0.08)$ and $8(0.1,0.21,0.04)$ by $4(0.1,0.21,0.04)$. Now the thresholded product is $\{(3(0.03,0.21,0.56), 2(0.05,0.27,0.42), 4(0.02,0.09,0.28), 4(0.15,0.49,0.08), 4(0.25,0.63,0.06)$, $4(0.1,0.21,0.04))\} \in 4-M\left(S_{1}\right)$.

$\left\{4-M\left(S_{1}\right), \times\right\}$ is a commutative neutrosophic multiset semigroup of infinite order and the multiplicity of any element cannot exceed 4 .

This semigroup is not a monoid and it has no special zero divisors or zero divisors or units.

Definition 12. 12 Let $n-M\left(S_{i}\right)=\left\{\right.$ collection of all multisets with entries from $S_{i}$ of at-most multiplicity $n ; 2 \leq n<\infty\}(1 \leq i<4) . n-M\left(S_{i}\right)$ under usual product, $\times$ is defined as the n-multiplicity NC semigroup, $1 \leq i \leq 4$.

In view of this we have the following theorem.

Theorem 11. Let $n-M\left(S_{i}\right)=\left\{t(x, y, z) \mid x, y, z \in S_{i} ; 1 \leq t \leq n\right\}$ be the n-multiplicity neutrosophic multisets $(1 \leq i \leq 4)$.

1. $n-M\left(S_{i}\right)$ is not closed under the binary operation ' + ' under usual addition, for $i=1,3$ and 4 .

2. $n-M\left(S_{i}\right)$ is a (n-multiplicity neutrosophic multiset) semigroup under the usual product for $i=1,2,3$ and 4 .

3. $\quad\left\{n-M\left(S_{i}\right), \times\right\}$ is a monoid for $i=3$ and 4 .

4. $\left\{n-M\left(S_{i}\right), \times\right\}$ has no special zero divisors if $S_{i}=S_{1}$ and $S_{3}$ but they have no non trivial idempotents. $S_{2}$ and special zero divisors and no non trivial idempotents, but $S_{4}$ has both non trivial special zero divisors and non trivial idempotents.

Proof. Proof of 1: If $A=\{(0.3,0.8,0.9)\}$ and $B=\{(0.4,0.3,0.1)\} \in \mathrm{n}-M\left(S_{i}\right) . A+B=\{(0.7,1.1$, $1.0)\} \notin \mathrm{n}-M\left(S_{i}\right)$ as $S_{i}$ when built using $S_{3}$ and $S_{4}$ and by example $4 \mathrm{n}-M\left(S_{1}\right)$. Only $M\left(S_{2}\right)$ is closed under addition.

Proof of 2: Since $\left(S_{i}, \times\right)$ is closed under product so is $\mathrm{n}-M\left(S_{i}\right)$ with replacing the numbers greater than $n$ by $n$ in the resultant product; $i=1,2,3$ and 4 are semigroups, hence the claim.

Proof of 3: As $(1,1,1) \in S_{3}$ and $S_{4}$ so is in $n-M\left(S_{3}\right)$ and $n-M\left(S_{4}\right)$ respectively so they are monoids.

Proof of 4: $\mathrm{n}-M\left(S_{i}\right)$ has no special zero divisors in case of $S_{1}$ and $S_{3}$. Finally $S_{i}=\{(x, y, z) \mid x, y, z \in$ $\left.S_{i}\right\}$, has zero divisors and special zero divisors in case of $S_{2}$ and $S_{4}$ for $i=2$ and 4 , and non trivial idempotents contributed by 0 's and 1 's only in case of $S_{4}$. Hence the theorem.

Example 5. Let $5-M\left(S_{2}\right)=$ Collection of all neutrosophic multisets which can occur at most 5-times that is the multiplicity is 5 with elements from $\left.S_{2}=\{(x, y, z) \mid x, y, z \in[0,1)\}\right\}$ Let $A=$ 
$4(0.2,0.5,0.7), 3(0.1,0.2,0.3), 5(0.3,0.1,0.2),(0.1,0.2,0.8) \in 5-M\left(S_{2}\right)$ We see the multiplicity of $(0.3,0.1$, $0.2)$ is 5 others are less than 5 .

Let $A=\{3(0.3,0.2,0), 4(0.5,0.6,0.9), 5(0.1,0.2,0.7)\}$ and $B=\{4(0.8,0.1,0.9), 2(0.6,0.6,0.6)\} \in$ $5-M\left(S_{2}\right)$. Now we first find $A \times B=\{5(0.24,0.02,0), 5(0.4,0.06,0.81), 5(0.08,0.02,0.63), 5(0.06,0.12$, $0.42)\} \in 5\left(M\left(S_{2}\right)\right)$.

$A+B=\{5(0.1,0.3,0.9), 5(0.9,0.8,0.6), 5(0.3,0.7,0.8), 5(0.9,0.3,0.6), 5(0.1,0.2,0.5), 5(0.7,0.8$, $0.3)\} \in 5-M\left(S_{2}\right)$. Addition is done modulo 1. However we have closure axiom to be true under + for elements in $S_{2}$ and in case of $\left.S_{1} ; 0 \notin S_{1}=(0,1)\right)$. This closure axiom is flouted.

If addition modulo 1 is done we have to see that 1 is not included in the interval and 0 is included in that interval so we need to have only closed open interval $[0,1)$. Under these two constraints only we can make $S_{2}$ as well as $M\left(S_{2}\right)$ and $n-M\left(S_{2}\right)$ as semigroups under addition modulo 1.

We can built strong structure only using the $[0,1)$.

Theorem 12. Let $n-M\left(S_{2}\right)=$ Collection of all multisets of $S$ built using $S_{2}=\{(x, y, z) \mid x, y, z \in[0,1)\}$ with multiplicity less than or equal to $n ; 2 \leq n \leq \infty$

$\left\{n-M\left(S_{2}\right), \times\right\}$ is a commutative neutrosophic multiset semigroup of infinite order and is not a monoid, $n-M\left(S_{2}\right)$ has infinite number of zero divisors.

Proof. If $\mathrm{A}$ and $B \in \mathrm{n}-M\left(S_{2}\right)$ we find $A \times B$ and update the multiplicities in $A \times B$ to be less than or equal to $\mathrm{n}$ so that $A \times B \in \mathrm{n}-M\left(S_{2}\right)$. by Theorem 11(2).

Clearly $(1,1,1) \notin \mathrm{n}-M\left(S_{2}\right)$ so is not a monoid.

Theorem 13. $B=\left\{n-M\left(S_{2}\right),+, \times\right\}$, the $n$-multiplicity multiset $N C$ is a commutative semiring of infinite order and has no unit, where $S_{2}=[0,1)$.

Proof. Follows from the fact $\left\{\mathrm{n}-M\left(S_{2}\right),+\right\}$ is a commutative semigroup under addition modulo 1, Theorem 11(1) and Theorem 12 and $\left\{\mathrm{n}-M\left(S_{2}\right), \times\right\}$ is a commutative semigroup under $\times$. Hence the claim.

\section{Discussions}

The main motive of this paper is to construct strong algebraic structures with two binary operations on the NC. Here we are able to get a NC commutative ring structure using the base interval as $[0,1)$. This will lead to future research of constructing Smarandache neutrosophic vector spaces and Smarandache neutrosophic algebraic codes using the same interval [0,1). Now using the same interval $[0,1)$, we construct multiset NC and n-multiset NC $2 \leq n<\infty$. On these we were able to built only neutrosophic multiset(n-multiplication set) commutative semiring structure. Now using these we can construct Smarandache multiset neutrosophic semi vector spaces which will be taken as future research. So this is significant first step to develop other strong structures and apply them to NC codes and NC cryptography.

\section{Conclusions}

In this paper, authors have made a study of NC on the 4-intervals $(0,1)(0,1],[0,1]$ and $[0,1)$. We define usual + and $\times$ on these intervals which is very different from the study taken so far. The main properties enjoyed by these NC semigroups are developed. Further of these intervals only the interval $[0,1)$ gives a nice algebraic structure viz an abelian group under usual addition modulo 1 , which in turn helps in constructing NC commutative ring under usual addition modulo 1 and product, the ring has infinite number of zero divisors, whereas all the other intervals are semigroups/monoids which are torsion free or weakly torsion free of infinite order under $\times$. Further in this paper we introduce the notion of multiset NC semigroups using these four intervals under product. Furthermore, the multiset 
NC forms a commutative semiring with zero divisors only when the interval $[0,1)$ is used. Finally we introduce n-multiplicity multiset using these NC. They are also semigroups which is torsion free or weakly torsion free under product.

For future research we will be using the product and addition modulo 1 in the place of min and max in Single Valued Neutrosophic Set (SVNS) and would compare the results with the existing ones when applied as SVNS models to real world problems.

Apart from all these we can use these NC, multiset NC and n-multiplicity multiset NC to built NC codes which is one of the applications to neutrosophic cryptography which will be taken up by the authors for future research.

Author Contributions: Conceptualization, V.W.B.; writing-original draft preparation, I.K.; writing-review and editing, F.S. All authors have read and agreed to the published version of the manuscript.

Funding: This research received no external funding.

Acknowledgments: We would like thanks the reviewers for their valuable suggestions.

Conflicts of Interest: The authors declare no conflict of interest.

\section{Abbreviations}

The following abbreviations are used in this manuscript:

SVNS Single Valued Neutrosophic Set

\section{References}

1. Herstein, I.N. Topics in Algebra; John Wiley \& Sons: Hoboken, NJ, USA, 2006.

2. Hall, M. The Theory of Groups; Courier Dover Publications: Mineola, NY, USA, 2018.

3. Howie, J.M. Fundamentals of Semigroup Theory; Clarendon Oxford: Oxford, UK, 1995.

4. Godin, T.; Klimann, I.; Picantin, M. On torsion-free semigroups generated by invertible reversible Mealy automata. In International Conference on Language and Automata Theory and Applications; Springer: Berlin, Germany, 2015; pp. 328-339.

5. East, J.; Egri-Nagy, A.; Mitchell, J.D.; Peresse, Y. Computing finite semigroups. J. Symb. Comput. 2019, 92, 110-155. [CrossRef]

6. Smarandache, F. A Unifying Field in Logics: Neutrosophic Logic. Neutrosophy, Neutrosophic Set, Probability, and Statistics; American Research Press: Rehoboth, DE, USA, 2000.

7. Smarandache, F.; Ali, M. Neutrosophic triplet group. Neural Comput Applic 2018, 29, 595-601. [CrossRef]

8. Kandasamy W.B., V.; Kandasamy, I.; Smarandache, F. Semi-Idempotents in Neutrosophic Rings. Mathematics 2019, 7, 507. [CrossRef]

9. Kandasamy W. B., V.; Kandasamy, I.; Smarandache, F. Neutrosophic Triplets in Neutrosophic Rings. Mathematics 2019, 7, 563. [CrossRef]

10. Kandasamy, W.V.; Kandasamy, I.; Smarandache, F. Neutrosophic Quadruple Vector Spaces and Their Properties. Mathematics 2019, 7, 758.

11. Saha, A.; Broumi, S. New Operators on Interval Valued Neutrosophic Sets. Neutrosophic Sets Syst. 2019, 28,10 .

12. Sahin, R.; Karabacak, M. A novel similarity measure for single-valued neutrosophic sets and their applications in medical diagnosis, taxonomy, and clustering analysis. In Optimization Theory Based on Neutrosophic and Plithogenic Sets; Elsevier: Amsterdam, The Netherlands, 2020; pp. 315-341.

13. Jain, A.; Nandi, B.P.; Gupta, C.; Tayal, D.K. Senti-NSetPSO: Large-sized document-level sentiment analysis using Neutrosophic Set and particle swarm optimization. Soft Comput. 2020, 24, 3-15. [CrossRef]

14. Wu, X.; Zhang, X. The Decomposition Theorems of AG-Neutrosophic Extended Triplet Loops and Strong AG-(1, 1)-Loops. Mathematics 2019, 7, 268. [CrossRef]

15. Ma, Y.; Zhang, X.; Yang, X.; Zhou, X. Generalized Neutrosophic Extended Triplet Group. Symmetry 2019, 11, 327. [CrossRef] 
16. Li, Q.; Ma, Y.; Zhang, X.; Zhang, J. Neutrosophic Extended Triplet Group Based on Neutrosophic Quadruple Numbers. Symmetry 2019, 11, 696. [CrossRef]

17. Ali, M.; Smarandache, F.; Khan, M. Study on the Development of Neutrosophic Triplet Ring and Neutrosophic Triplet Field. Mathematics 2018, 6, 46. [CrossRef]

18. Smarandache, F. Neutrosophic Perspectives: Triplets, Duplets, Multisets, Hybrid Operators, Modal Logic, Hedge Algebras. And Applications; EuropaNova: Bruxelles, Belgium, 2017.

19. Kandasamy, W.V.; Ilanthenral, K. Smarandashe Special Elements in Multiset Semigroups; EuropaNova ASBL: Brussels, Belgium, 2018.

20. Forsberg, L. Multisemigroups with multiplicities and complete ordered semi-rings. Beitr Algebra Geom 2017, 58, 405-426. [CrossRef]

21. Kandasamy, W.V. Smarandache Semirings, Semifields, And Semivector Spaces. Smarandache Notions J. 2002, 13, 88.

22. Blizard, W.D. The development of multiset theory. Mod. Log. 1991, 1, 319-352.

(C) 2020 by the authors. Licensee MDPI, Basel, Switzerland. This article is an open access article distributed under the terms and conditions of the Creative Commons Attribution (CC BY) license (http:/ / creativecommons.org/licenses/by/4.0/). 